\title{
Nouvelles applications des insaponifiables et des lipides de synthèse d'origine végétale en dermocosmétique
}

\author{
Nathalie PICCARDI ${ }^{1}$ \\ Nicole BROUTIN ${ }^{1}$ \\ Sébastien GARNIER ${ }^{1}$ \\ Jacques LEGRAND ${ }^{1}$ \\ Jean-Christophe CHOULOT $^{1}$ \\ Bernard CHADOUTAUD ${ }^{2}$ \\ Philippe MSIKA ${ }^{1}$ \\ ${ }^{1}$ Laboratoires Expanscience, \\ Centre de recherche et développement, \\ Rue des 4 Filles, \\ 28230 Epernon \\ $<$ npiccardi@expanscience.com> \\ ${ }^{2}$ BC Consultant, \\ 7, avenue Marcel Langer, \\ 31000 Toulouse
}

\begin{abstract}
Lipid chemistry is the main know-how of the research and development Center of Laboratoires Expanscience. Our paper will be focused on this field with special emphasize on three different techniques molecular distillation, bio-enzymatic reaction and green chemistry. Each technique will be illustrated by an active molecule, namely Soline ${ }^{\circledR}, 5-\alpha$ Avocuta $^{\circledR}$, Cycloceramide $^{\circledR}$. The process, composition as well as the efficiency of the product will be presented. These data are illustrated by numerous patents and scientific communications.
\end{abstract}

Key words: lipids, molecular distillation, green chemistry, biotechnology, pharmacology, clinical evaluation, cosmetic applications
La chimie des lipides constitue depuis de nombreuses années un pôle d'excellence pour les Laboratoires Expanscience, avec un savoir-faire historique dans le domaine de l'oléochimie de I'avocat, et plus particulièrement de sa fraction insaponifiable, obtenue par distillation moléculaire, avec une maîtrise totale de la filière du fruit jusqu'au produit fini.

La distillation moléculaire est un procédé physique (évaporation contrôlée sous vide poussé) qui a pour but d'extraire, de purifier, de concentrer, de désodoriser des molécules thermosensibles et de haut poids moléculaire telles que les vitamines, les stérols, les caroténoïdes. La distillation moléculaire est réalisée sous un vide poussé (100 000 à $10^{6}$ plus faible que la pression atmosphérique), et dans des conditions de température optimale. À ce titre, les Laboratoires Expanscience ont amélioré ce procédé en couplant à cette technologie celle du chauffage à induction [1]. Appliqué aux huiles végétales, ce procédé permet notamment de concentrer la fraction insaponifiable dans le distillat, sans en altérer les molécules essentielles qui la constituent.

En parallèle, le Centre de R\&D a développé de nouveaux savoir-faire dans le domaine du génie bioenzymatique et de la synthèse (" chimie verte»). Ces différentes technologies, associées à l'objectivation pharmacologique et clinique, nous ont permis de mettre au point des molécules naturelles et innovantes pour des applications dermocosmétiques ciblées.

\section{Soline ${ }^{\circledR}$}

\section{Origine et composition}

Soline ${ }^{\circledR}$ (nom $\mathrm{INCl}$ : helianthus annus (sunflower) seed oil unsaponifiables) est une fraction insaponifiable originale obtenue par distillation moléculaire, à partir d'une huile de tournesol sélectionnée. Ce traitement permet de concentrer dix fois la fraction insaponifiable de cette huile. Soline ${ }^{\circledR}$ est composé de triglycérides comportant $90 \%$ d'acides gras insaturés, $20 \%$ d'acide oléique et $60 \%$ d'acide linoléique, de $5 \%$ de phytostérols, et de $1 \%$ de vitamine E naturelle (90\% d' $\alpha$-tocophérol).

\section{Activité pharmacologique et clinique}

La fonction barrière de la peau est liée à la présence du stratum corneum, couche la plus superficielle de l'épiderme, composée de cellules aplaties et sans noyau, les cornéocytes. L'étanchéité de ce «mur de briques» est assuré par un ciment intercellulaire composé de lipides spécifiques (cholestérol, cholestérol sulfate, acides gras libres et céramides). Les céramides, lipides-clés de l'épiderme, représentent 40 à $50 \%$ de ces lipides, le cholestérol 20 à $25 \%$, les acides gras libres 15 à $25 \%$ et le cholestérol sulfate de 5 à $10 \%$.

Les céramides jouent un rôle-clé dans la régulation de la fonction barrière de la peau (étanchéité). Le ratio cholestérol/cholestérol sulfate régule quant à lui la cohésion entre les cornéo- cytes, et donc le phénomène de desquamation, ainsi que la fluidité du film lipidique.

De nombreux désordres dermatologiques sont associés à un trouble de la barrière cutanée. Ainsi, le vieillissement cutané est associé à une modification de la fonction barrière de la peau, consécutive à une réduction de la synthèse des lipides-clés de l'épiderme [2]. La dermatite atopique est associée à une diminution de la fonction barrière de la peau, corrélée à une diminution de la synthèse des lipides épidermiques, et notamment des céramides [3].

Restaurer la fonction barrière et pallier le déficit de la synthèse des lipides épidermiques représentent donc un challenge incontournable pour l'industrie cosmétique. À ce titre, nous avons montré ex vivo (explant de peau humaine) qu'une émulsion contenant $2 \%$ de Soline ${ }^{\circledR}$ stimulait positivement et spécifiquement les lipides-clés de l'épiderme (cérébrosides, cholestérol, céramide), sans modification de la quantité totale de lipides (figure 1). Dans cette étude, le placebo testé en parallèle était sans effet [4].

De nombreuses études cliniques ont confirmé l'intérêt de Soline ${ }^{\circledR}$ dans la prise en charge des peaux sèches et à tendance atopiques [5].

\section{Évaluations cliniques et biométrologiques chez l'adulte}

Une étude en double aveugle a été réalisée chez 20 volontaires adultes atopiques, de sexe féminin, âgés de 20 à 28 ans, présentant une 
peau sèche à très sèche, porteuses de légères desquamations. Le produit testé est une émulsion contenant $2 \%$ de Soline ${ }^{\circledR}$, en une application unique ou en deux applications par jour pendant quatre semaines. Des mesures biométrologiques ainsi qu'une évaluation clinique ont été réalisées.

On note immédiatement (après 1 et 3 heures) et sur le long terme (24 $\mathrm{h}$ après l'application), une nette amélioration de I'hydratation des couches supérieures de l'épiderme (figure 2). Selon l'examen pratiqué par le directeur de l'étude, l'aspect de la peau est également amélioré (figure 3) avec une diminution statistiquement significative de la rugosité (- $52 \%)$, de la sécheresse $(-54 \%)$ et de la desquamation (- $54 \%)$. Ces résultats sont également confirmés par les volontaires ayant participé au test. Le microrelief est restructuré (figure 4). Un effet relipidant immédiat est également mis en évidence (sébumètre). Par ailleurs, les applications ont été très bien tolérées.

\section{Évaluation clinique chez l'enfant atopique}

Une étude a été réalisée auprès de 227 enfants atopiques en poussées légères à modérées (lésions non suintantes, sans dermocorticoïdes), sous contrôle dermatologique et pédiatrique. Le produit testé $\left(2 \%\right.$ de Soline $\left.{ }^{\circledR}\right)$ a été appliqué à raison de 2 fois par jour, pendant 30 jours.

Ce test a permis de mettre en évidence une amélioration très significative des signes cliniques liés à l'atopie : sécheresse $(-88 \%)$, desquamation $(-84 \%)$, prurit $(-80 \%)$ et érythème $(-80 \%)$.

Les résultats des tests ex vivo et in vivo sont donc corrélés, Soline ${ }^{\circledR}$ active spécifiquement la synthèse des lipides-clés de l'épiderme et améliore significativement la fonction barrière de la peau : c'est le concept de Relipidation Active ${ }^{\circledR}$.

\section{5 a-Avocuta ${ }^{\circledR}[6]$}

L'oléochimie de l'avocat sconstitue depuis 30 ans un pôle d'excellence des Laboratoires Expanscience, avec une maîtrise totale de la filière du fruit au produit fini. De nombreux brevets dans les domaines de la pharmacie et de la cosmétique illustrent aujourd'hui ce savoir-faire. En combinant notre connaissance des fractions lipidiques de l'avocat avec un procédé de génie bioenzymatique, nous avons mis au point le $5 \alpha$-Avocuta ${ }^{\circledR}$ (nom INCI : butyl avocadate), un mélange complexe et spécifique d'esters butyliques d'avocat.

\section{Origine et composition}

Le procédé d'obtention du $5 \alpha$-Avocuta ${ }^{\circledR}$ comprend une première étape de purification de I'huile vierge d'avocat par distillation moléculaire, suivie d'une réaction de transestérifica-

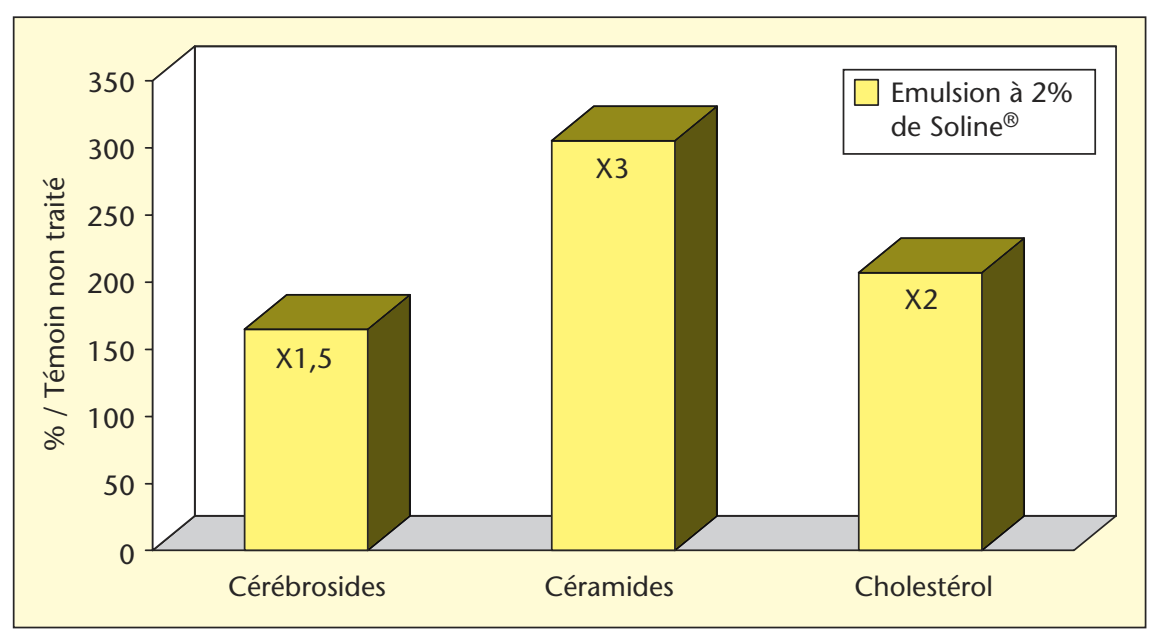

Figure 1. Stimulation de la synthèse des lipides épidermiques dans un modèle d'explant cutané.

Effet d'une émulsion contenant $2 \%$ de Soline $e^{\circledast}$ (une seule application, 24 heures de contact). Les résultats sont exprimés en \% par rapport à la peau non traitée.

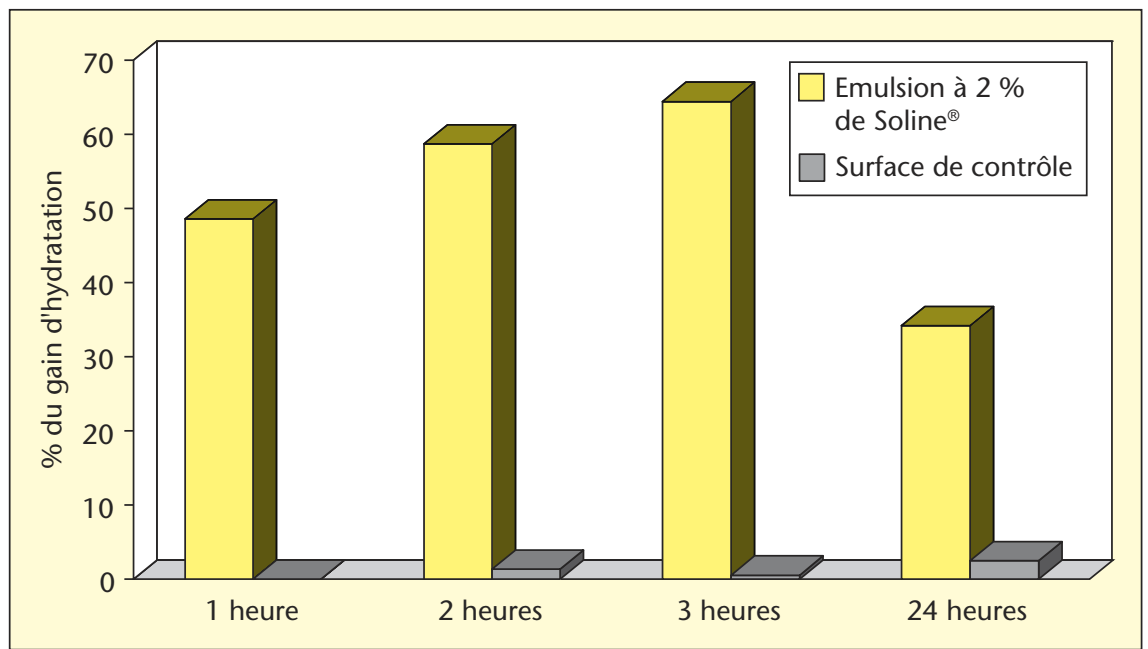

Figure 2. Mesure de l'hydratation cutanée par cornéométrie. Effet d'une émulsion contenant $2 \%$ de Soline ${ }^{\circledast}$.

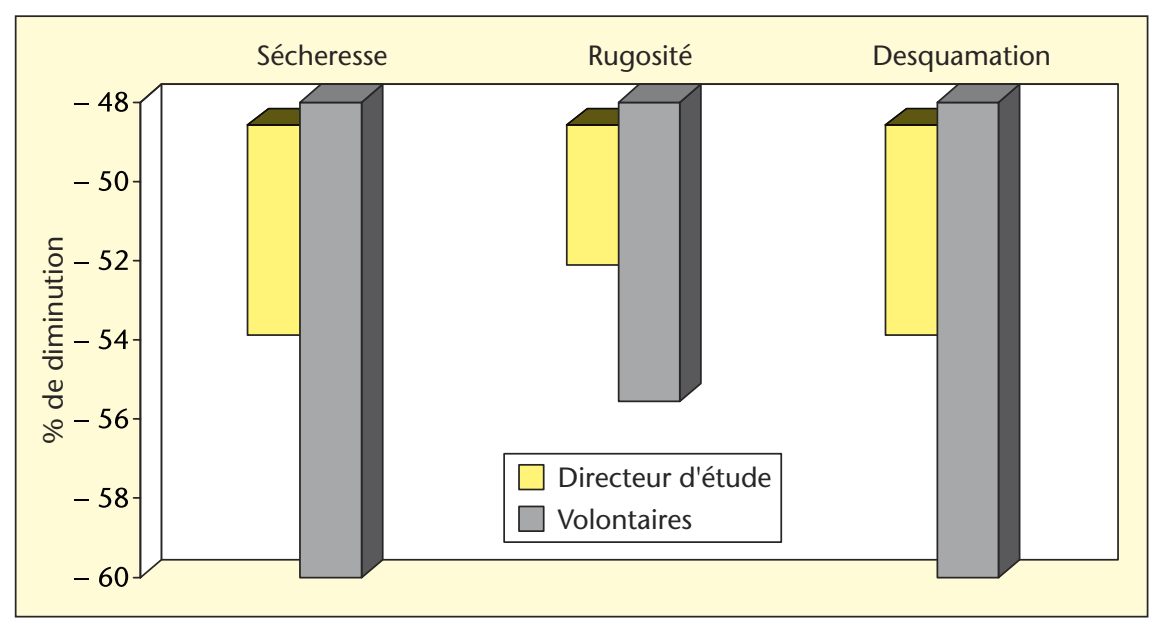

Figure 3. Évolution de l'aspect de la peau après quatre semaines d'application (deux fois par jour) d'une émulsion contenant $2 \%$ de Soline ${ }^{\circledast}$ 


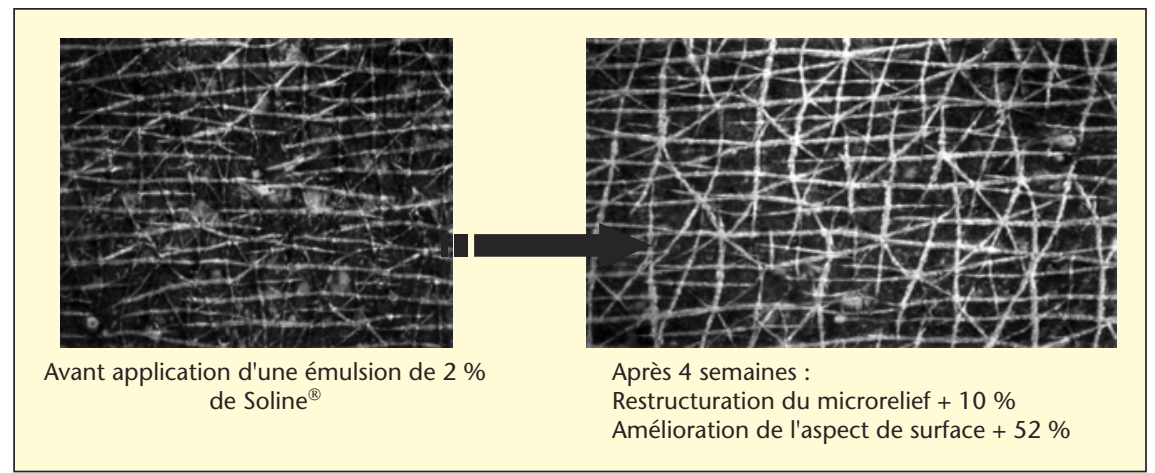

Figure 4. Evolution du microrelief cutané après quatre semaines d'application (deux fois par jour) d'une émulsion contenant $2 \%$ de Soline ${ }^{\circledast}$

tion en présence de butanol et d'un catalyseur enzymatique (figure 5). Enfin, les esters butyliques d'avocat sont purifiés par distillation moléculaire.

\section{Activité pharmacologique et clinique}

La peau, et plus particulièrement les glandes sébacées sont activement impliquées dans la production des androgènes $[7,8]$. La métabolisation de la testostérone en 5-alpha dihydrotestostérone (5-alpha DHT), le plus puissant androgène tissulaire, est médiée via l'enzyme 5-alpha réductase. Deux types de 5-alpha réductase ont été isolés chez I'homme, le type I et le type II. Le type I est la forme prédominante au niveau de la peau, et est exprimée dans les glandes sébacées, les glandes sudorales et dans l'épiderme [9]. Son activité est maximale au niveau des glandes sébacées du visage et du scalp $[10,11]$.

Le sébum est composé d'un mélange de lipides non polaires, dont la plupart sont synthétisés de novo par les glandes sébacées. L'augmentation de l'excrétion du sébum est par ailleurs un facteur-clé dans la physiopathologie de l'acné [12].

Le $5 \alpha$-Avocuta ${ }^{\circledR}$ a donc été spécifiquement développé afin d'optimiser son activité en tant qu'inhibiteur de la 5-alpha réductase.
Nous avons montré que le $5 \alpha$-Avocuta ${ }^{\circledR}$ inhibait de manière significative et dosedépendante, l'activité de la 5-alpha réductase dans un modèle de fibroblastes cutanés en culture [13]. In vitro, cette inhibition atteint $49 \%$ pour une concentration de $0,01 \%$ en $5 \alpha$-Avocuta ${ }^{\circledR}$ (figure 6).

L'efficacité clinique de cet actif a été validée dans le cadre de l'hyperséborrhée du cuir chevelu et du visage [14]. Les deux études réalisées avaient pour objectif de valider l'efficacité et la tolérance du $5 \alpha$-Avocuta ${ }^{\circledR}$, dans des conditions réelles d'utilisation à travers des évaluations qualitatives et quantitatives, réalisées à la fois par les dermatologues et les volontaires.

\section{Efficacité clinique sur hyperséborrhée du cuir chevelu}

Cette étude multi-centrique a réuni 27 volontaires adultes qui ont utilisé pendant 3 semaines (une application tous les deux jours) un shampoing contenant $1 \%$ de $5 \alpha$-Avocuta ${ }^{\circledR}$. L'efficacité du shampoing a été évaluée par :

- la mesure des scores lipidiques en début et fin d'étude (Sebufix $\mathrm{F} 16^{\circledR}$ );

- un examen visuel pratiqué par le dermatologue en début et fin d'étude ;

- une analyse d'image des patchs pour 13 volontaires (Skinvisometer SV60 ${ }^{\circledR}$, CK, Allemagne).
Nous avons ainsi mis en évidence que le 5 -Avocuta ${ }^{\circledR}$, formulé à $1 \%$, réduisait de manière significative la production de sébum (- $34 \%)$, améliorait l'état du cuir chevelu, avec notamment une diminution des picotements, du prurit, de l'érythème et des pellicules (figure 7). Les résultats de l'analyse d'image des patchs ont confirmé l'efficacité du $5 \alpha$-Avocuta ${ }^{\circledR}$ en termes de réduction de la production de sébum (figure 8), avec sur l'ensemble des 13 cas étudiés, une diminution du nombre de tâches lipidiques (-137), une diminution de la surface lipidique cumulée $(-69 \%)$, et une diminution du ratio surface lipidique/surface totale (- 7,8 points). De plus, il répond parfaitement aux critères des consommateurs présentant une hyperséborrhée du cuir chevelu, et son efficacité est soulignée par une excellente tolérance.

\section{Évaluation clinique sur l'hyperséborrhée du visage}

23 volontaires de sexe féminin, présentant une hyperséborrhée du visage ( $87 \%$ avec des antécédents d'acné) ont appliqué pendant trois semaines (deux applications par jour) une crème contenant $2 \%$ de $5 \alpha$-Avocuta ${ }^{\circledR}$.

L'efficacité du traitement a été évaluée par la mesure des scores lipidiques au niveau du front (évaluation visuelle, Sebufix $\mathrm{F} 16^{\circledR}$ ), et par le biais de deux évaluations l'une réalisée par les dermatologues, l'autre par les volontaires.

Au terme des trois semaines, nous avons enregistré une diminution de $30 \%$ de la production de sébum au niveau du front (examen visuel des patchs). Cet effet a également été visualisé par les dermatologues au niveau du nez $(-33 \%)$, des joues $(-30 \%)$ et du menton (- $31 \%)$. L'efficacité du produit a également été soulignée par les volontaires.

Ainsi, après 3 semaines, le $5 \alpha$-Avocuta ${ }^{\circledR}$, formulé à $2 \%$, permet de réduire la production excessive de sébum au niveau du visage, et améliore l'aspect de la peau. Les applications topiques répétées du $5 \alpha$-Avocuta ${ }^{\circledR}$ sont par ailleurs bien tolérées.

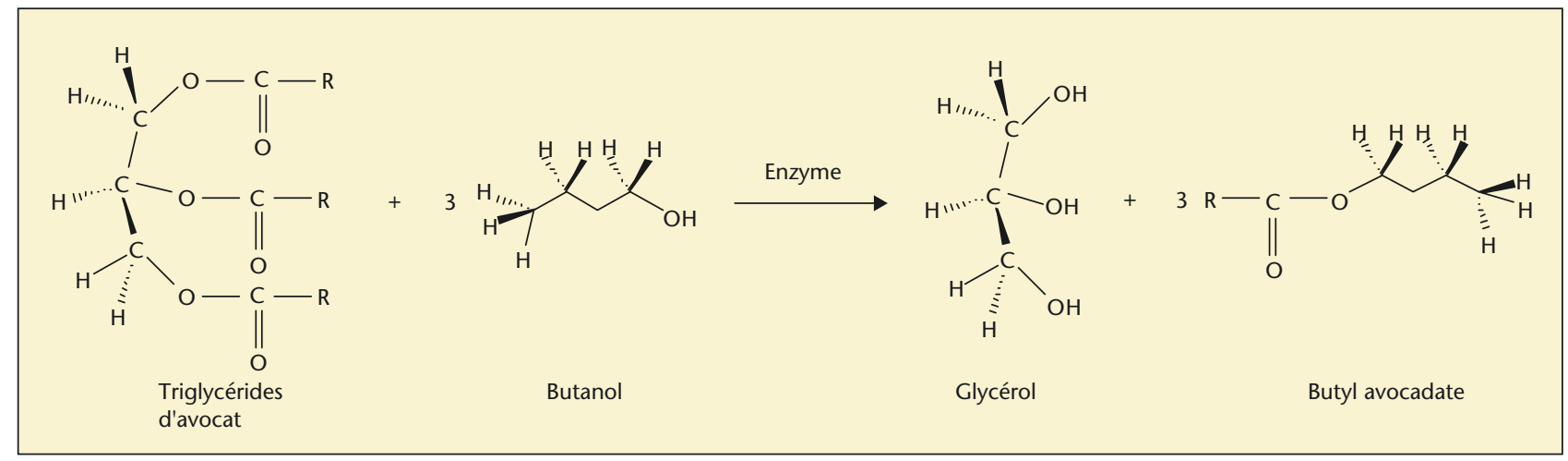

Figure 5. Réaction de transestérification de I'huile d'avocat par le butanol. 


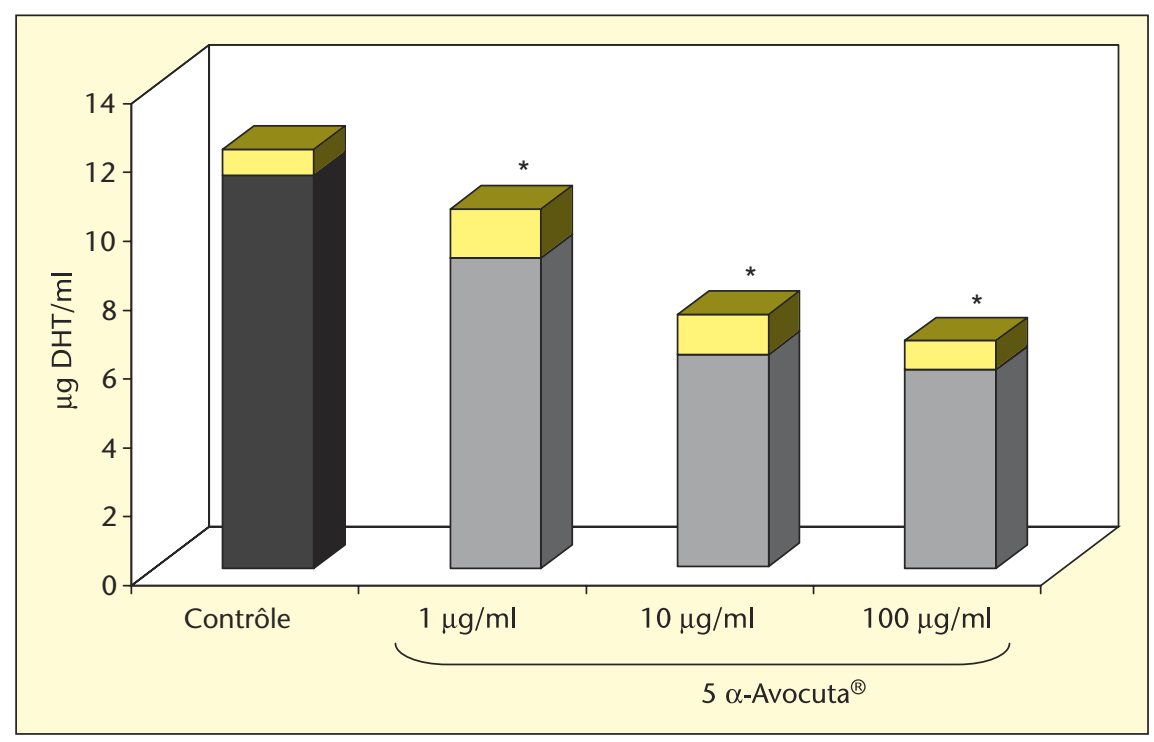

Figure 6. Inhibition de l'activité 5-alpha réductase de type 1 par le $5 \alpha$-Avocuta ${ }^{\circledR}$.

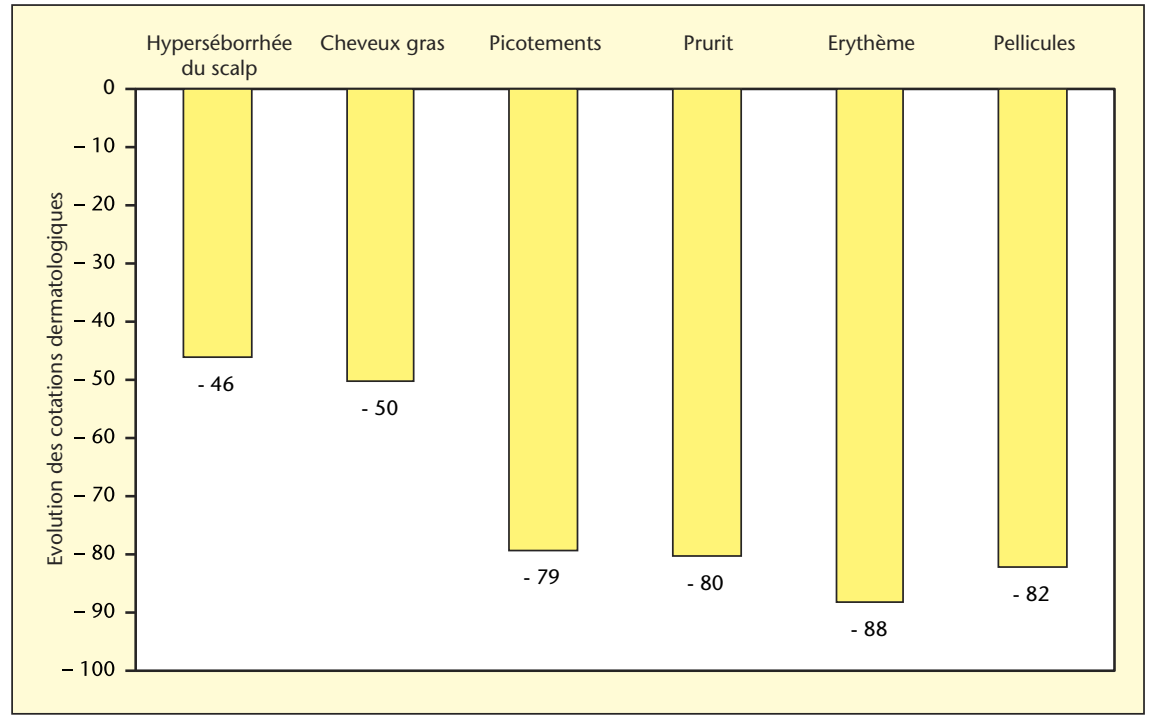

Figure 7. Le $5 \alpha$-Avocuta ${ }^{\circledR}$ permet de faire évoluer de manière très favorable l'état du cuir chevelu.

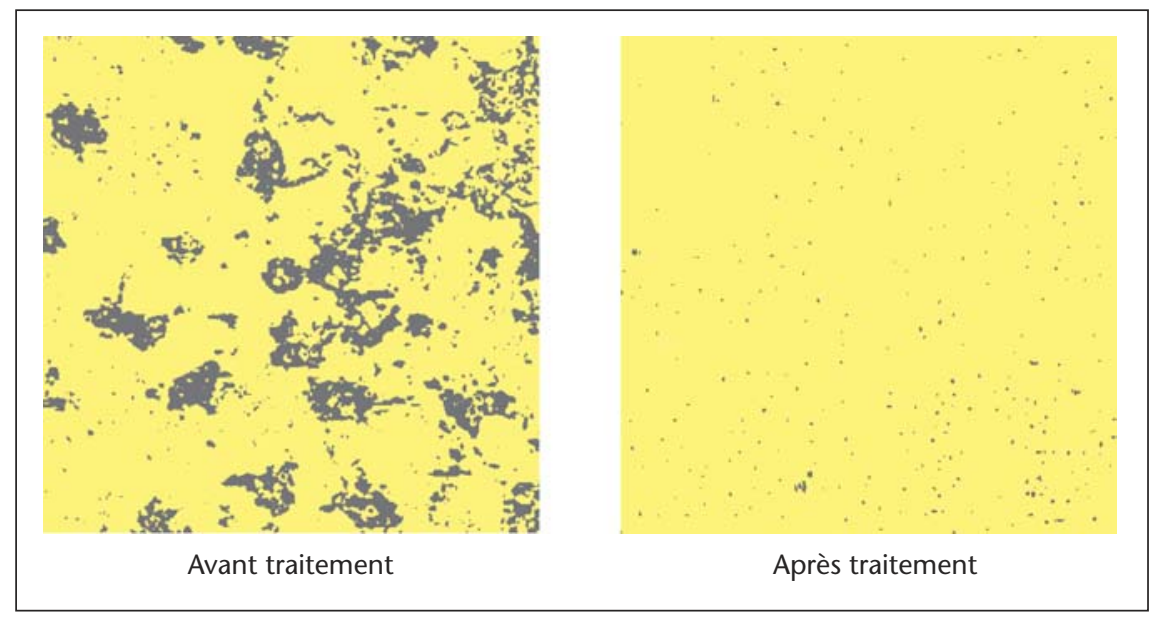

Figure 8. Visualisation de la diminution de la sécrétion de sébum après utilisation du shampoing contenant $1 \%$ de $5 \alpha$-Avocuta ${ }^{\circledR}$. Analyse d'image des patchs Sebufix $F 16^{\circledR}$ (exemple représentatif : cas $n^{\circ} 12$ ).
Les esters d'huile d'avocat qui composent le $5 \alpha$-Avocuta ${ }^{\circledR}$ constituent donc une réponse originale aux problèmes d'hyperséborrhée.

\section{Le Cyclocéramide ${ }^{\circledR}[15]$}

\section{Origine et composition}

La collaboration avec une équipe du CNRS (Laboratoire de catalyse en chimie organique, UMR 6503-CNRS-ESIP, Poitiers) a permis de mettre au point, le Cyclocéramide ${ }^{\circledR}$ (nom $\mathrm{INCl}$ : undecyl dimethyl oxazoline), combinaison d'un acide gras d'origine végétale (huile de coco) avec un amino alcool spécifique en présence d'un catalyseur hétérogène recyclable. Ce procédé peut être avantageusement classé au rang de la «chimie verte » de part son caractère respectueux de l'environnement (rejets quasi nuls) et la non-utilisation de solvants et composés chimiques corrosifs. Le Cyclocéramide ${ }^{\circledR}$ est ensuite purifié par distillation moléculaire. Cette molécule, appartient à la famille des 2-oxazolines (figure 9).

\section{Activité pharmacologique}

Cette molécule a été conçue afin d'optimiser sont efficacité vis-à-vis de l'inhibition de l'activité PKC (protéine kinase $\mathrm{C}$ ), notamment dans le but de limiter la réaction inflammatoire. En effet, la PKC apparaît comme un facteur-clé de la production des médiateurs de l'inflammation, notamment de l'interleukine-1 [16].

Nous avons mis en évidence que le Cyclocéramide ${ }^{\circledR}$ inhibait la PKC- $\beta 2$ humaine in vitro (cellules $S f 9$, enzyme recombinante) avec une $I_{50}$ de $33 \mu \mathrm{M}$.

D’autre part, le Cyclocéramide ${ }^{\circledR}$ présente des activités anti-inflammatoires in vitro. Cette molécule est en effet capable de limiter le relarguage de I'IL-1 $\beta$ dans le milieu de culture de kératinocytes préalablement soumis au SDS (sodium dodécyl sulfate) (figure 10).

Suite à des travaux, réalisés sur cellules, et initiés avec l'équipe Inserm 346, nous avons mis en évidence, en collaboration avec l'équipe Inserm 503, les propriétés anti-inflammatoires du Cyclocéramide ${ }^{\circledR}$ dans un modèle d'allergie de contact primaire chez la souris [17]. Grâce à ce modèle, nous avons montré que l'application topique de Cyclocéramide ${ }^{\circledR}(0,1 \%$, une application/jour pendant trois jours consécutifs) réduisait de manière très significative la réaction inflammatoire non spécifique induite par une dose irritante de DNFB (dinitro-fluoro benzène) (figure 11) [18].

Le Cyclocéramide ${ }^{\circledR}$ a par ailleurs démontré son efficacité clinique chez l'homme dans le cadre d'études en ouvert et en double aveugle contre 


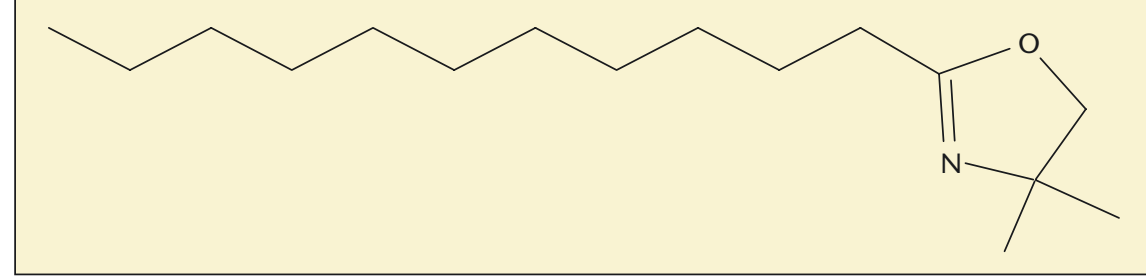

Figure 9. Cyclocéramide ${ }^{\circledR}$ (2-undécyl-4,4-diméthyl-2-oxazoline).

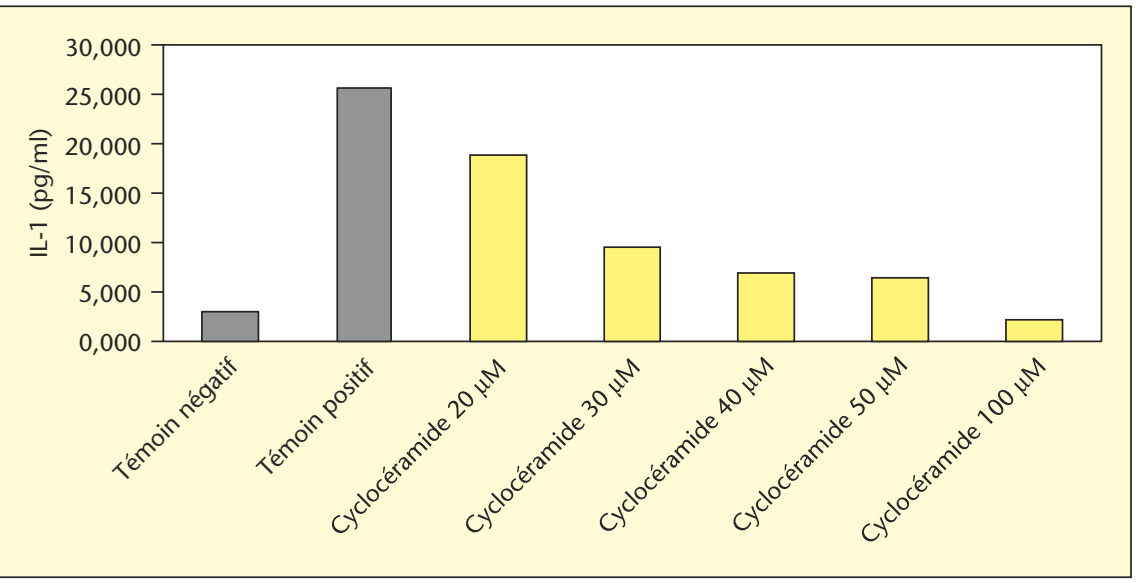

Figure 10. Effet du Cyclocéramide ${ }^{\circledR}$ sur le relarguage de I'IL-1 $\beta$ dans un modèle de kératinocytes irrités.

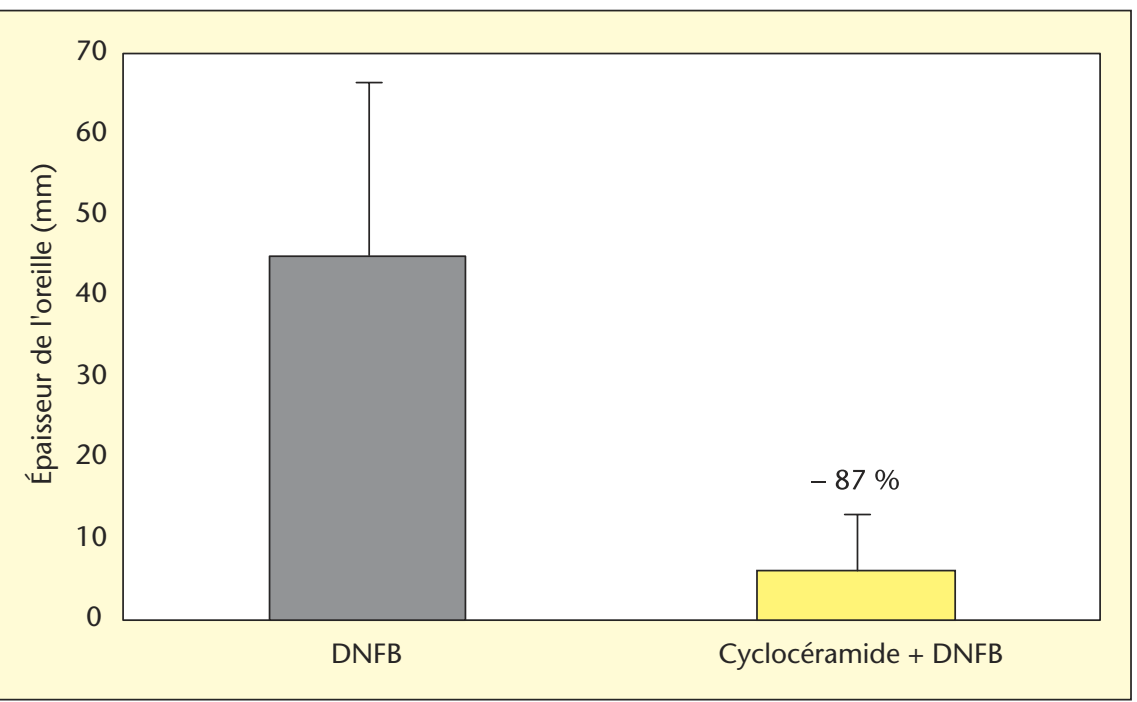

Figure 11. Effet du Cyclocéramide ${ }^{\circledR}$ sur la réaction inflammatoire induite chez la souris par application de DNFB.

placebo chez des personnes présentant une hyper-réactivité cutanée $[19,20]$.

Le Cyclocéramide ${ }^{\circledR}$, inhibiteur de PKC, a démontré son efficacité dans la modulation de la réaction inflammatoire modélisée in vitro et in vivo, ainsi que dans la prise en charge de I'hyper-réactivité cutanée chez l'homme.

Ces exemples reflètent l'évolution du savoirfaire du Centre de recherche et développement des Laboratoires Expanscience, dans le domaine de la chimie des lipides, et plus particulièrement dans la rationalisation de leur composition et/ou structure dans le but d'optimiser leurs propriétés pharmacologiques et leurs activités cliniques. Fruits de cette stratégie, Soline ${ }^{\circledR}$, le Cyclocéramide ${ }^{\circledR}$ et le 5- $\alpha$ Avocuta $^{\circledR}$ sont des lipides naturels originaux, à l'excellente tolérance. Leur efficacité démontrée dans le cadre d'études ouvertes ou en double aveugle contre placebo font de ces molécules des actifs de choix permettant de répondre aux exigences grandissantes de l'industrie cosmétique.

\section{RÉFÉRENCES}

1. BREVET FR 9014518.

2. ELIAS PM, GHADIALLY R. The aged epidermal permeability barrier: basis for functional abnormalities. Clin Geriatr Med 2002 ; 18(1) : 103-20.

3. PROKSCH E, JENSEN JM, ELIAS PM. Skin lipids and epidermal differentiation in atopic dermatitis. Clin Dermatol $2003 ; 21$ : 134-44.

4. BREVET WO0121150.

5. PICCIRILLIA, CHOULOT JC, PICCARDI N, MSIKA P. Sunflower oil oleodistillate for atopy treatment : an in vitro and clinical evaluation. I Eur Acad Dermatol Venereol 2000 ; 14(suppl 1) : 318-9.

6. BREVET WO0152837.

7. ZOUBOULIS CC. Human skin : an independent peripheral endocrine organ. Horm Res 2000 ; $54: 230-42$.

8. FRITSCH M, ORFANOS CE, ZOUBOULIS CC. Sebocytes are the key regulators of androgen homeostasis in human skin. I Invest Dermatol $2001 ; 116: 793-800$.

9. LUU-THE V, SUGIMOTO Y, PUY L, ET AL. Characterization, expression, and immunohistochemical localization of 5a-reductase in human skin. J Invest Dermatol $1994 ; 102$ : $221-6$.

10. THIBOUTOT D, HARRIS G, ILES V, ET AL. Activity of the type I 5a-reductase exhibits regional differences in isolated sebaceous glands and whole skin. J Invest Dermatol $1995 ; 105$ : 20914.

11. CHEN W, ZOUBOULIS CC, FRITSCH M, ET AL. Evidence of heterogeneity and quantitative differences of the type I $5 \alpha$-reductase expression in culture human skin cells. Evidence of its presence in melanocytes. / Invest Dermatol $1998 ; 110: 84-9$.
12. CUNLIFFE W]. Acne. London: Martin Dunitz, 1989.

13. PICCIRILLI A, LEGRAND I, MSIKA P. Development of new 5-alpha reductase inhibitors. I Invest Dermatol $2001 ; 117(2)$ : 388-552. 
14. PICCARDIN, PICCIRILLIA, CHOULOT JC, CHADOUTAUD B, MSIKA P. Pharmacological and clinical activities of 5 alpha-Avocuta : application for the management of androgenic disorders. I Invest Dermatol 2004; 123(2): A69.

15. BREVET WO03055463.

16. KIONG Y, HARMON CS. Intreleukine-1 beta is differentially expressed by human dermal papilla cells in response to PKC activation and is a potent inhibitor of human hair follicle growth in organ culture. I Interferon Cytokines Res $1997 ; 17(3)$ : 151-7.
17. SAINT-MÉZARD P, KRASTEVA M, CHAVAGNAC C. Afferent and efferent phases of allergic contact dermatitis (ACD) can be induced after a single skin contact with haptens: evidence using a mouse model of primary ACD. I Invest Dermatol $2003 ; 120(4): 641-7$.

18. BÉRARD F, SAINT-MÉZARD P, RODET K, ET AL. Blockade of skin dendritic cell migration by topical application of MMP and PKC inhibitors is associated with inhibition of allergic contact dermatitis in vivo. I Invest Dermatol 2004; 122(2) : A57 ; abstract 339.
19. CHADOUTAUD B, CHOULOT JC, PICCARDI N, MSIKA P. Clinical evaluation of a cream containing MMP and PKC inhibitors on sensitive and irritated skin. J Invest Dermatol 2003; 121(1): 734 ; abstract 0365).

20. CHADOUTAUD B, CHOULOT JC, PICCARDI N, MSIKA P, NICOLAS JF. Double blind randomised trial evaluation of a new product containing MMP and PKC inhibitors in cutaneous hyper-sensitivity. I Invest Dermatol 2004; 122(2) : A126 ; (abstract 754). 